\title{
General proof of the entropy principle for self-gravitating fluid in $f(R)$ gravity
}

\author{
Xiongjun Fang, ${ }^{a}$ Minyong $\mathrm{Guo}^{b}$ and Jiliang Jing ${ }^{a, 1}$ \\ ${ }^{a}$ Department of Physics and Key Laboratory of Low Dimensional Quantum Structures and \\ Quantum Control of Ministry of Education, Hunan Normal University, \\ Changsha, Hunan 410081, P.R. China \\ ${ }^{b}$ Department of Physics, Beijing Normal University, \\ Beijing 100875, P.R. China \\ E-mail: fangxj@hunnu.edu.cn, minyongguo@mail.bnu.edu.cn, \\ jljing@hunnu.edu.cn
}

ABSTRACT: The discussions on the connection between gravity and thermodynamics attract much attention recently. We consider a static self-gravitating perfect fluid system in $f(R)$ gravity, which is an important theory could explain the accelerated expansion of the universe. We first show that the Tolman-Oppenheimer-Volkoff equation of $f(R)$ theories can be obtained by thermodynamical method in spherical symmetric spacetime. Then we prove that the maximum entropy principle is also valid for $f(R)$ gravity in general static spacetimes beyond spherical symmetry. The result shows that if the constraint equation is satisfied and the temperature of fluid obeys Tolmans law, the extrema of total entropy implies other components of gravitational equations. Conversely, if $f(R)$ gravitational equation hold, the total entropy of the fluid should be extremum. Our work suggests a general and solid connection between $f(R)$ gravity and thermodynamics.

Keywords: Classical Theories of Gravity, Cosmology of Theories beyond the SM

ArXiv EPrint: 1512.05454

\footnotetext{
${ }^{1}$ Corresponding author.
} 


\section{Contents}

1 Introduction 1

2 TOV equations obtained by two different methods 3

2.1 Classical dynamical method 3

2.2 Thermodynamical method 4

3 Maximum entropy principle for general static spacetime in $f(R)$ gravity 6

4 Summary and discussions $\quad 9$

$\begin{array}{ll}\text { A Detail calculation of } \delta \rho & 10\end{array}$

$\begin{array}{lr}\text { B Calculation of } P_{1}^{a b} \text { and } P_{2}^{a b} & 13\end{array}$

\section{Introduction}

Black holes are mysterious and important objects which have been studied for a long time. The mathematical analogy between laws of black hole mechanics and the ordinary laws of thermodynamics leads to the discovery of black hole thermodynamics [1]. Hawking proved that the black hole behaves like a black body with a temperature which is proportional to its surface gravity, and one quarter of the horizon area is the entropy of the black hole $[2,3]$. Since then, black hole thermodynamics has been widely studied, and people believe that it could help us to catch sight of some important fundamental theories.

It is generally believed that the gravity equation is the basic equation of nature. However, Jacobson put forward a new point of view that the Einstein equation is an equation of state [4]. Over the years this point has been accepted by more and more people. R. Cai proved that applying the first law of thermodynamics to the apparent horizon of a Friedmann-Robertson-Walker universe, one could derive the Friedmann equations describing the dynamics of the universe [5]. Then Verlinde considered gravity could be explained as an entropic force [6]. And Bravetti presented an example of spacetimes effectively emerging from the thermodynamic limit over an unspecified microscopic theory without any further assumptions [7]. All these discussion showed that the gravity equation may not be the basic assumption but the thermodynamic relation is.

In fact, before the establish of black hole thermodynamics, Cocke [8] proposed maximum entropy principle for self-gravitating fluid. In contrast to black hole, the local thermodynamic quantities of self-gravitating fluid, such as entropy density $s$, energy density $\rho$ and local temperature $T$, are well defined. The presence of gravity only affects the distribution of these local quantities. Roughly speaking, there are two different ways to approach the 
distribution of the self-gravitating fluid system. One way is called the dynamical method, and the other way is called the thermodynamical method. In dynamical method, using Einstein's equation and applying conservation of matter, people could obtain the distribution of the fluid. But in thermodynamical method, using only constrain Einstein equation and the ordinary thermodynamical relation, and admits some natural assumption, then the fluid could be configured such that its total entropy attains a maximum value. For a spherical radiation system, Sorkin, Wald and Zhang [9] showed that if the total entropy be an extremum and the Einstein constraint equation holds, then the Tolman-OppenheimerVolkoff (TOV) equation of hydrostatic equilibrium can be derived. Gao [10] generalized SWZ's work to arbitrary perfect fluid in static spherical spacetime and got the TOV equation for the fluid. This issue has been further explored in the past years [11-14]. Recently, series of general proofs of the maximum entropy principle in the case of static spacetime without the spherical symmetry has been completed, included Einstein-Maxwell theory and Lovelock theory [15-18].

However, whether the entropy principle is valid for $f(R)$ gravity is not clear. $f(R)$ gravity is a natural generalization of Einstein gravity. The motivation of modifying generalization of GR comes from high-energy physics for adding higher order invariants to the gravitational action, as well as comes from cosmology and astrophysics for seeking generalizations of GR. As an important modified theories, $f(R)$ theories can explain the accelerated expansion of the universe because it contains the $\alpha R^{2}$ terms [19]. $f(R)$ gravity comes about by a straightforward generalization of the Lagrangian in the Einstein action to become a general function of Ricci scalar $R$, i.e.,

$$
S=\frac{1}{2 \kappa} d^{4} x \sqrt{-g} f(R),
$$

where $f$ as a series expansion of $R$

$$
f(R)=\sum_{n=-\infty}^{n=\infty} \alpha_{n} R^{n} .
$$

There are two advantages in $f(R)$ gravity [20,21]. First, $f(R)$ actions are simple enough to be deal with, but sufficiently general to encapsulate some of the basic characteristics of higher-order gravity. Second, there are some reasons to believe that $f(R)$ gravity is unique among higher-order gravity theories which can avoid Ostrogradski instability. Thus it is interesting to discuss the maximum entropy principle in this generalized gravity theories.

In section II, we discuss the maximum entropy in spherical static spacetime. TOV equation constrains the structure of a spherically symmetric body of isotropic material which is in static gravitational equilibrium. We first briefly review how to obtain the modified TOV equation of $f(R)$ gravity by traditional dynamical method. And then in addition to the constraint equation, we only make use of the ordinary thermodynamical relations to derive the same TOV equation in $f(R)$ gravity. These two different methods obtained the same results shows that the maximum entropy in spherical static spacetime in $f(R)$ theories is valid.

In section III, with the help of Tolman's law, which was also used in $[13,15,18]$, we extend the entropy principle to general static spacetimes without spherical symmetry 
condition. It should be noticed that, the total entropy $S$ appears to depend on $\delta h_{a b}$ and $\delta R$ (See appendix A). Fixing the induced metric $h_{a b}$ and scalar curvature $R$ and their first derivatives on the boundary, in order to allow us to use integration by parts and drop the boundary terms. And it also shows that the components of $\delta R$ vanishes automatically. So the vanishing of the components of $\delta h_{a b}$ just gives the spatial components of gravitational equation of $f(R)$ theories. The derivation of this section shows that the maximum entropy is also valid in $f(R)$ theories without spherical symmetry.

Throughout our discussion, symbol $a, b, c$ represent the abstract index notation, $\nabla_{a}$ and $D_{a}$ are the covariant derivative associated with the metric of spacetime and the covariant derivative associated with the induced metric $h_{a b}$, respectively, and $\square \equiv \nabla^{a} \nabla_{a}$.

\section{TOV equations obtained by two different methods}

\subsection{Classical dynamical method}

As for the issue of the distribution of fluid, dynamical method is a classical one, and is widely used. First we briefly review how to get TOV equation in $f(R)$ gravity by dynamical method [22]. In 4-dimensional spacetime the action of the $f(R)$ gravity can be expressed as

$$
S=\frac{1}{2 \kappa} \int d^{4} x \sqrt{-g} f(R)+S_{M}
$$

where $\kappa \equiv 8 \pi$, and $S_{M}$ is the action term of perfect fluid matter. Variation with respect to the metric gives

$$
f_{R}(R) R_{a b}-\frac{1}{2} f(R) g_{a b}-\left[\nabla_{a} \nabla_{b}-g_{a b} \square\right] f_{R}(R)=8 \pi T_{a b},
$$

where

$$
T_{a b}=-\frac{2}{\sqrt{-g}} \frac{\delta S_{M}}{\delta g^{a b}}
$$

and $f_{R}(R) \equiv \partial f / \partial R$.

For a static perfect fluid system with spherical symmetries in the $f(R)$ gravity, the line element of the spacetime can be written as

$$
d s^{2}=-e^{2 \phi(r)} d t^{2}+e^{2 \lambda(r)} d r^{2}+r^{2}\left(d \theta^{2}+\sin ^{2} \theta d \phi^{2}\right) .
$$

The energy-momentum tensor of the perfect fluid is

$$
T_{a b}=\rho u_{a} u_{b}+p h_{a b}
$$

where $\rho$ is the matter density and $p$ is the pressure, and in our chosen coordinate we have $u^{a}=-e^{\phi(r)}(d t)_{a}$. The components of the field equations become

$$
\begin{aligned}
8 \pi T_{t}^{t}=8 \pi \rho= & \frac{f_{R}(R)}{r^{2}}+\frac{e^{-2 \lambda}\left(2 r \lambda^{\prime}-1\right)}{r^{2}} f_{R}(R)+\frac{1}{2} f(R) \\
& -\frac{R}{2} f_{R}(R)-e^{-2 \lambda} f_{R}^{\prime \prime}(R)+e^{-2 \lambda}\left(\lambda^{\prime}-\frac{2}{r}\right) f_{R}^{\prime}(R),
\end{aligned}
$$


and

$$
\begin{aligned}
8 \pi T_{r}{ }^{r}=8 \pi p= & -\frac{f_{R}(R)}{r^{2}}+\frac{e^{-2 \lambda}\left(1+2 r \phi^{\prime}\right)}{r^{2}} f_{R}(R)-\frac{1}{2} f(R) \\
& +\frac{R}{2} f_{R}(R)+e^{-2 \lambda} \frac{2+r \phi^{\prime}}{r} f_{R}^{\prime},
\end{aligned}
$$

where' ${ }^{\prime} \equiv d / d r$.

To get the TOV equation, we consider Bianchi identity, which leads to energymomentum conservation equation $\nabla_{a} T_{b}^{a}=0$. The radial component of the conservation equation gives

$$
p^{\prime}=-(\rho+p) \phi^{\prime} .
$$

If we assume

$$
e^{-2 \lambda}=1-\frac{2 m}{r}
$$

Then the modified TOV equations take the following convenient form

$$
\begin{gathered}
8 \pi p+\frac{2 m}{r^{3}} f_{R}(R)+\frac{f(R)}{2}-\frac{R}{2} f_{R}(R)-\frac{2(r-2 m)}{r^{2}} f_{R}^{\prime}(R) \\
=-\frac{r-2 m}{r}\left(\frac{2 f_{R}(R)}{r}+f_{R}^{\prime}(R)\right) \frac{p^{\prime}}{p+\rho} .
\end{gathered}
$$

Notice that if the Lagrangian degenerate to Einstein theory, i.e. $f(R)=R$, eq. (2.10) will reduce to the well-known TOV equation in ref. [23].

\subsection{Thermodynamical method}

In previous subsection, we have shown how to get the TOV equation in $f(R)$ gravity by using dynamical method. Generally, to get the TOV equation, one has to use radial-radial component (eq. (2.7)) of the gravitational equation. Now, we shall derive TOV equation in $f(R)$ gravity by using thermodynamical method. We only use conservation equation (eq. (2.6)) and the extrema of total entropy, admits the condition of the variation of total particle number vanish in static spacetime, to derive TOV equation of $f(R)$ gravity. It implies that the maximum entropy principle contains part of information of gravitational equations in $f(R)$ gravity.

The perfect fluid system satisfies the familiar first law

$$
d S=\frac{1}{T} d E+\frac{P}{V} d V-\frac{\mu}{T} d N
$$

where $S, E, N$ represent the total entropy, energy, and particle number within the volume $V$. Their density variables are denoted by $s, \rho$ and $n$, respectively. Applying eq. (2.11) to a unit volume, it is easy to find

$$
d s=\frac{1}{T} d \rho-\frac{\mu}{T} d n,
$$

and the integrated form which be called the Gibbs-Duhem relation

$$
s=\frac{p+\rho-\mu n}{T} .
$$


Now we apply the maximum entropy principle to this self-gravitating perfect fluid in $f(R)$ gravity. In spherical symmetry case, the total entropy and the total particle number from $r=0$ to $r=r_{0}$ can be written as [10]

$$
\begin{aligned}
& S=4 \pi \int_{0}^{r_{0}} s(r)\left[1-\frac{2 m(r)}{r}\right]^{-1 / 2} r^{2} d r \\
& N=4 \pi \int_{0}^{r_{0}} n(r)\left[1-\frac{2 m(r)}{r}\right]^{-1 / 2} r^{2} d r .
\end{aligned}
$$

The variation of total particle number vanishes, which means $\delta N=0$, is a desired condition in maximum entropy principle. Following the standard method of Lagrange multipliers, the equation of variation becomes

$$
\delta S+\lambda \delta N=0
$$

Define the "total Lagrangian" by

$$
L\left(m, m^{\prime}, n\right)=s\left(\rho\left(m, m^{\prime}\right), n\right)\left(1-\frac{2 m(r)}{r}\right)^{-\frac{1}{2}} r^{2}+\lambda n(r)\left(1-\frac{2 m(r)}{r}\right)^{-\frac{1}{2}} r^{2} .
$$

So the constrained Euler-Lagrange equation is given by

$$
\begin{aligned}
\frac{\partial L}{\partial n} & =0, \\
\frac{d}{d r} \frac{\partial L}{\partial m^{\prime}} & =\frac{\partial L}{\partial m} .
\end{aligned}
$$

Eq. (2.18) yields,

$$
\frac{\partial s}{\partial n}=\lambda
$$

By using thermodynamic relation we have

$$
\mu=\lambda T \text {. }
$$

Now consider eq. (2.19), after some calculation, we have

$$
\frac{\partial L}{\partial m}=\frac{1}{8 \pi T}\left(\frac{2}{r} f_{R}^{\prime \prime}+\frac{3}{r^{2}} f_{R}^{\prime}\right) r^{2}\left(1-\frac{2 m}{r}\right)^{-1 / 2}+(n \lambda+s) r\left(1-\frac{2 m}{r}\right)^{-3 / 2}
$$

and

$$
\frac{\partial L}{\partial m^{\prime}}=\frac{1}{8 \pi T}\left(\frac{2}{r^{2}} f_{R}(R)+\frac{1}{r} f_{R}^{\prime}(R)\right) r^{2}\left(1-\frac{2 m}{r}\right)^{-1 / 2} .
$$

By substituting these results into eq. (2.19), together with eqs. (2.13) and (2.21), we could obtain the Euler-Lagrangian equation of $m$ in an explicit form

$$
\begin{aligned}
\frac{T^{\prime}}{8 \pi T^{2}} & \left(\frac{2}{r^{2}} f_{R}(R)+\frac{1}{r} f_{R}^{\prime}(R)\right) r^{2} \\
= & \frac{1}{8 \pi T}\left(-\frac{4}{r^{3}} f_{R}(R)-\frac{1}{r^{2}} f_{R}^{\prime}(R)+\frac{2}{r^{2}} f_{R}^{\prime}(R)+\frac{1}{r} f_{R}^{\prime \prime}(R)\right) r^{2} \\
& +\frac{1}{8 \pi T}\left(\frac{2}{r^{2}} f_{R}(R)+\frac{1}{r} f_{R}^{\prime}(R)\right)\left(2 r+r m^{\prime}-5 m\right)\left(1-\frac{2 m}{r}\right)^{-1} \\
& -\frac{1}{8 \pi T}\left(\frac{2}{r} f_{R}^{\prime \prime}+\frac{3}{r^{2}} f_{R}^{\prime}\right) r^{2}-\frac{p+\rho}{T} r\left(1-\frac{2 m}{r}\right)^{-1} .
\end{aligned}
$$


The constraint eq. (2.21) yield

$$
\mu^{\prime}=\lambda T^{\prime}
$$

Considering the fundamental thermodynamic relation

$$
d p=s d T+n d \mu,
$$

it follows that

$$
p^{\prime}=s T^{\prime}+n \mu^{\prime}=\frac{T^{\prime}}{T}(\rho+p) .
$$

Thus we obtain the equation

$$
\begin{aligned}
\frac{p^{\prime}}{\rho+p} & \left(\frac{2}{r^{2}} f_{R}(R)+\frac{1}{r} f^{\prime}(R)\right) r^{2}\left(1-\frac{2 m}{r}\right) \\
= & \left(-\frac{4}{r^{3}} f_{R}(R)+\frac{1}{r^{2}} f_{R}^{\prime}+\frac{1}{r} f_{R}^{\prime \prime}\right) r^{2}\left(1-\frac{2 m}{r}\right)+\left(\frac{2}{r^{2}} f_{R}+\frac{1}{r} f_{R}^{\prime}\right)\left(2 r+r m^{\prime}-5 m\right) \\
& -\left(\frac{2}{r} f_{R}^{\prime \prime}(R)+\frac{3}{r^{2}} f_{R}^{\prime}(R)\right) r^{2}\left(1-\frac{2 m}{r}\right)-8 \pi p r-8 \pi \rho r
\end{aligned}
$$

By substituting eq. (2.6) into eq. (2.28), finally we get

$$
\begin{aligned}
& -\frac{p^{\prime}}{\rho+p}\left(\frac{2}{r^{2}} f_{R}(R)+\frac{1}{r} f^{\prime}(R)\right) r^{2}\left(1-\frac{2 m}{r}\right) \\
& \quad=8 \pi p+\frac{f(R)}{2}+\left(\frac{2 m}{r^{3}}-\frac{R}{2}\right) f_{R}(R)-\frac{2(r-2 m)}{r^{2}} f_{R}^{\prime}(R) .
\end{aligned}
$$

This TOV equation in $f(R)$ gravity is exactly the same as eq. (2.10).

It is necessary to point out that, under the condition of total particle number fixed, we only use the maximum entropy principle and the time-time component gravitational equation. No other assumptions are needed. It tells us that in spherical symmetry case, the extrema of entropy contain part of information of gravitational equation. It shows that the maximum entropy principle is valid in spherical symmetric case in $f(R)$ theories. And this is a direct evidence for the fundamental relationship between gravitation and thermodynamics.

\section{Maximum entropy principle for general static spacetime in $f(R)$ grav- ity}

In previous sections, we got TOV equation by using dynamical and thermodynamic methods, respectively. But all these discussions based on static spherical symmetry condition. What we want to know is that whether the maximum entropy principle is valid for general static spacetime in $f(R)$ gravity.

Consider a general static spacetime $\left(M, g_{a b}\right)$ and $\Sigma$ as a three dimensional hypersurface denoting a moment of the static observers. Let $C$ be a region on $\Sigma$ with a boundary $\bar{C}$ and $h_{a b}$ be the induced metric on $\Sigma$, and $R$ be the scalar curvature of spacetime, respectively. We assume that the Tolman's law holds in $C$, like [15], which means that the local temperature $T$ of the fluid satisfies

$$
T \chi=T_{0},
$$


where $\chi$ is the red-shift factor for static observers and $T_{0}$ is a constant which can be viewed as the red-shift temperature of the fluid. Without loss of generality, we take $T_{0}=1$ in Tolman's law. Assume that the constraint equation is satisfied in $C$. We also need $h_{a b}, R$ and their first derivatives are fixed on $\bar{C}$, which allow us to use integration by parts and drop the boundary term. Then, it can be proved that the other components of gravitational equation are implied by the extrema of the total fluid entropy for all variations of data in $C$.

We consider a general perfect fluid as discussed in previous section. The induced metric on $\Sigma$ is given by

$$
h_{a b}=g_{a b}+u_{a} u_{b}
$$

The stress-energy tensor $T_{a b}$ takes the form of eq. (2.5). And from conservation law $\nabla_{a} T^{a b}=0$, we can show that

$$
\nabla_{a} p=-(\rho+p) A_{a}
$$

where $A_{a}$ is the four-acceleration of the observer. Since

$$
u^{a}=\frac{\xi^{a}}{\chi}
$$

where $\xi^{a}$ is the Killing vector. One can show that

$$
A_{a}=\nabla_{a} \chi / \chi
$$

and thus

$$
\nabla_{a} p=-(\rho+p) \nabla_{a} \chi / \chi
$$

Meanwhile, the local first law can also be expressed in the form

$$
d p=s d T+n d \mu
$$

Comparing with eqs. (3.6) and (3.7), and applying the Tolman's law eq. (3.1) and the Gibbs-Duhem relation eq. (2.13), we finally have

$$
\mu \chi=\mu / T=\text { constant }
$$

If we choose energy density $\rho$ and particle number density $n$ as two independent thermodynamical variables. Then in static spacetime, the total entropy $S$ is an integral of the entropy density $s$ over the region $C$ on $\Sigma$,

$$
S=\int_{C} \sqrt{h} s(\rho, n) .
$$

where $h$ is the determinant of $h_{a b}$. Thus, the variation of the total entropy can be written as

$$
\delta S=\int_{C} s \delta \sqrt{h}+\sqrt{h} \delta s .
$$

Using the local first law of thermodynamics,

$$
T d s=d \rho-\mu d n
$$


we find

$$
\begin{aligned}
\delta S & =\int_{C} s \delta \sqrt{h}+\sqrt{h}\left(\frac{\partial s}{\partial \rho} \delta \rho+\frac{\partial s}{\partial n} \delta n\right) \\
& =\int_{C} s \delta \sqrt{h}+\sqrt{h}\left(\frac{1}{T} \delta \rho-\frac{\mu}{T} \delta n\right) .
\end{aligned}
$$

Notice that $\mu / T$ is constant, which can be taken out of the integral. It is natural to require the total number of particles

$$
N=\int_{C} \sqrt{h} n
$$

to be invariant, which leads to

$$
\int_{C} \sqrt{h} \delta n=-\int_{C} n \delta \sqrt{h}
$$

So we have

$$
\begin{aligned}
\delta S & =\int_{C}\left(s+\frac{n \mu}{T}\right) \delta \sqrt{h}+\sqrt{h} \frac{1}{T} \delta \rho \\
& =\int_{C} \frac{\rho+p}{T} \delta \sqrt{h}+\sqrt{h} \frac{1}{T} \delta \rho .
\end{aligned}
$$

Using [24]

$$
\delta \sqrt{h}=\frac{1}{2} \sqrt{h} h^{a b} \delta h_{a b}
$$

and denoting

$$
\delta S=\int_{C} \delta L
$$

we have

$$
\delta L=\frac{1}{2} \frac{\rho+p}{T} \sqrt{h} h^{a b} \delta h_{a b}+\sqrt{h} \frac{1}{T} \delta \rho .
$$

After some calculation, we get the exactly express of the term $\sqrt{h} \delta \rho / T$ in appendix A. By substituting eq. (A.25) into eq. (3.18), we have

$$
\begin{aligned}
\delta L= & \frac{\rho+p}{2 T} \sqrt{h} h^{a b} \delta h_{a b}+\delta L_{\rho} \\
= & \frac{\rho+p}{2 T} \sqrt{h} h^{a b} \delta h_{a b}+\frac{\sqrt{h}}{16 \pi} D^{b} D^{a}\left(\chi f_{R}(R)\right) \delta h_{a b}-\frac{\sqrt{h}}{16 \pi} D_{c}\left[h^{a b} D^{c}\left(\chi f_{R}(R)\right)\right] \delta h_{a b} \\
& -\frac{\sqrt{h}}{16 \pi} f_{R}(R) \chi R^{(3) a b} \delta h_{a b}-\frac{\sqrt{h}}{8 \pi} D^{a} \chi\left(D^{b} f_{R}(R)\right) \delta h_{a b}+\frac{\sqrt{h}}{16 \pi} h^{a b} D_{c}\left(\chi D^{c} f_{R}(R)\right) \delta h_{a b} .
\end{aligned}
$$

So far, we only applied the constraint equation $G_{00}=T_{00}$. This shows explicitly that $\delta S$ is determined only by the variation of $h_{a b}$. Since $\delta S=0$ in our assumption, it is clear shows that $\delta L=0$. Together with eq. (A.9) and Tolman's law eq. (3.1), it is easily find

$$
\begin{aligned}
\frac{8 \pi p h^{a b}}{T} \delta h_{a b}= & \frac{h^{a b}}{T} \delta h_{a b}\left[h^{a c} h^{b d} R_{c d} f_{R}(R)-\frac{1}{2} f(R) h^{a b}-h^{a c} h^{b d} \nabla_{c} \nabla_{d} f_{R}(R)+h^{a b} \square f_{R}(R)\right] \\
& -\frac{1}{2}\left(P_{1}^{a b}+P_{2}^{a b}\right) \delta h_{a b},
\end{aligned}
$$


where $P_{1}$ denotes the terms parallel to $h^{a b}$,

$$
\begin{aligned}
P_{1}^{a b}= & \chi h^{a b}\left[f_{R}(R) R_{c d} u^{c} u^{d}+\frac{1}{2} f(R)-u^{c} u^{d} \nabla_{c} \nabla_{d} f_{R}(R)-\square f_{R}(R)\right] \\
& -\chi \frac{1}{2} f(R) h^{a b}+h^{a b} \chi \square f_{R}(R)-h^{a b} D_{c} D^{c}\left(\chi f_{R}(R)\right)+h^{a b} D_{c}\left(\chi D^{c} f_{R}(R)\right),
\end{aligned}
$$

and $P_{2}$ denotes the other terms,

$$
\begin{aligned}
P_{2}^{a b}= & \chi\left[f_{R}(R) R_{c d} h^{a c} h^{b d}-h^{a c} h^{b d} \nabla_{c} \nabla_{d} f_{R}(R)\right]+D^{b} D^{a}\left(\chi f^{\prime}(R)\right) \\
& -f^{\prime}(R) \chi R^{(3) a b}-2 D^{a} \chi\left(D^{b} f_{R}(R)\right) .
\end{aligned}
$$

We show that $P_{1}^{a b}$ and $P_{2}^{a b}$ are vanish in appendix B. So eq. (3.20) gives the projection of gravitational equation

$$
8 \pi p=h^{a c} h^{b d} R_{c d} f_{R}(R)-\frac{1}{2} f(R) h^{a b}-h^{a c} h^{b d} \nabla_{c} \nabla_{d} f_{R}(R)+h^{a b} \square f_{R}(R) .
$$

In the above proof, under the fixed boundary condition, we only used the constraint eq. (A.9) to derive eq. (3.19). By applying the extrema of entropy $\delta S=0$, we obtained the spatial components of gravitational equation. It is easy to check that the proof is reversible. From the spatial components of gravitational equation, one can show $\delta L=0$ in eq. (3.19).

\section{Summary and discussions}

The TOV equation is an important equation for self-gravitating system. In this paper, we first briefly review how to get modified TOV equation in $f(R)$ gravity by dynamical method. By applying both time-time and radial-radial gravitational equations, and the radial component constraint equation, one can show that the modified TOV equation in $f(R)$ gravity is given by eq. (2.10). Then, by applying the maximum entropy principle to a general self-gravitating fluid, we have obtained the same TOV equation of hydrostatic equilibrium by thermodynamical method. We should point out that, to derive the TOV equation by the extrema of the total entropy $S$, we only need the constraint equation and fix the total particle number of the system. A comparison of these two different processes indicates that the maximum entropy principle is valid in spherical symmetry case in $f(R)$ theories. It should be noted that particle number conservation is equivalent to the radial part of the field equation under some conditions, including the time part of the field equation, thermodynamics law, the maximum of entropy and Tolman's law.

We also prove that the maximum entropy principle of perfect fluid is valid in the case without any symmetry on spacelike hypersurface. Selecting a region arbitrarily, consider the fluid obeys Tolman's law and the constraint equation is satisfied in this region. Then fix the induced metric $h_{a b}$ and scalar tensor $R$ and their first derivative on the boundary of selected region. Our calculation shows that $\delta S$ appears to depend on $\delta h_{a b}$ and $\delta R$, and the component of $\delta R$ always vanish. It shows that the variation of total entropy in this region is exactly linear to $\delta h_{a b}$. The assumption of $\delta S=0$ means the coefficient of $\delta h_{a b}$ vanish, which imply that the other components of gravitational equation are satisfied. 
Notice that under the conformal transformation $\widetilde{g}_{a b}=f_{R} g_{a b}$, and defining a scalar field

$\phi=\sqrt{\frac{3}{16 \pi}} \ln f_{R}, f(R)$ theories could be recast as the general relativity coupled with non minimal matter,

$$
\widetilde{G}_{a b}=\frac{8 \pi T_{a b}}{e^{4 \sqrt{\frac{\pi}{3}}} \phi}+8 \pi \widetilde{T}_{a b}^{\phi}
$$

with

$$
\widetilde{T}_{a b}^{\phi}=\widetilde{\nabla}_{a} \phi \widetilde{\nabla}_{b} \phi-\widetilde{g}_{a b}\left(\frac{1}{2} \widetilde{\nabla}_{c} \phi \nabla^{c} \phi+V\right) .
$$

where the scalar field $\phi$ is determined by the curvature of spacetime, then it relates to the metric. So $\delta \phi$ or $\delta \widetilde{g}_{a b}$ should be transformed to $\delta g_{a b}$. This implies that using the conformal transformation technique can also carry out this calculation.

Till now, the maximum entropy principle has been set up in series of theories. Although it is unclear whether this principle could be used for a general Lagrangian of gravity theories which needs further study, we consider that there may exist some deeper connection between gravity theories and thermodynamics.

\section{Acknowledgments}

We thank S. Gao and X. He for many helpful discussions. This work is supported by the National Natural Science Foundation of China under Grant No. 11475061; the SRFDP under Grant No. 20114306110003; the Open Project Program of State Key Laboratory of Theoretical Physics, Institute of Theoretical Physics, Chinese Academy of Sciences, China Grant No. Y5KF161CJ1. Xiongjun Fang is also supported by China Postdoctoral Science Foundation funded project.

\section{A Detail calculation of $\delta \rho$}

In this appendix, we will show the detailed calculation of $\delta \rho$ in eq. (3.18). First, note that the extrinsic curvature of $\Sigma$, is defined by

$$
\hat{B}_{a b}=h_{a}{ }^{c} h_{b}{ }^{d} \nabla_{d} u_{c} .
$$

It is straightforward to show

$$
\hat{B}_{a b}=\nabla_{b} u_{a}+A_{a} u_{b},
$$

where $A_{a}$ is the four-acceleration of the observer. And in static spacetime we have

$$
\hat{B}_{a b}=0
$$

so we get a very helpful formula in our calculation,

$$
\nabla_{b} u_{a}=-A_{a} u_{b}
$$

Then, we show [24] that the curvature $R_{a b c}^{(3)} d$ of hypersurface $\Sigma$ is related to the spacetime curvature $R_{a b c}{ }^{d}$ by

$$
R_{a b c}^{(3) d}=h_{a}{ }^{f} h_{b}{ }^{g} h_{c}{ }^{k} h_{j}{ }^{d} R_{f g k}{ }^{j}
$$


From this definition, it is easy to find

$$
R_{a b}^{(3)}=R_{a b}+R_{a e b}^{l} u^{e} u_{l}+R_{f b} u^{f} u_{a}+R_{a k} u^{k} u_{b}+u_{a} u_{b} R_{f k} u^{f} u^{k},
$$

and

$$
R^{(3)}=R+2 R_{a b} u^{a} u^{b} .
$$

Meanwhile, from eq. (A.6) we also have

$$
R^{(3) a b}=R_{c d}^{(3)} h^{a c} h^{b d}=R^{a b}+R_{a e b}^{l} u^{e} u_{l} h^{a c} h^{b d} .
$$

From eq. (2.5), one can easily get the Hamiltonian constraint of the theory,

$$
8 \pi \rho=u^{a} u^{b} R_{a b} f_{R}(R)+\frac{1}{2} f(R)-u^{a} u^{b} \nabla_{a} \nabla_{b} f_{R}(R)-\square f_{R}(R) .
$$

Using eq. (A.7), the variation of $\rho$ is

$$
\begin{aligned}
8 \pi \delta \rho= & \delta\left[u^{a} u^{b} R_{a b} f_{R}(R)+\frac{1}{2} f(R)-u^{a} u^{b} \nabla_{a} \nabla_{b} f_{R}(R)-\square f_{R}(R)\right] \\
= & \frac{1}{2} \delta\left[\left(R^{(3)}-R\right) f_{R}(R)\right]+\frac{1}{2} \delta f(R)-\delta\left[u^{c} u^{d} \nabla_{c} \nabla_{d} f_{R}(R)\right]-\delta\left[\square f_{R}(R)\right] \\
= & \frac{1}{2} f_{R}(R) \delta R^{(3)}+\frac{1}{2}\left(R^{(3)}-R\right) f_{R R}(R) \delta R \\
& -\delta\left[u^{c} u^{d} \nabla_{c} \nabla_{d} f_{R}(R)\right]-\delta\left[\square f_{R}(R)\right],
\end{aligned}
$$

where we denote $f_{R R}(R) \equiv \frac{\partial^{2} f}{\partial R^{2}}$, and we use $\delta f(R)=f_{R}(R) \delta R$. So $\delta \rho$ term in eq. (3.18) becomes

$$
\begin{aligned}
\delta L_{\rho}= & \sqrt{h} \frac{\delta \rho}{T} \\
= & \frac{\sqrt{h}}{16 \pi T} f_{R}(R) \delta R^{(3)}+\frac{\sqrt{h}}{16 \pi T}\left(R^{(3)}-R\right) f_{R R}(R) \delta R \\
& -\frac{\sqrt{h}}{8 \pi T} \delta\left[u^{a} u^{b} \nabla_{a} \nabla_{b} f_{R}(R)\right]-\frac{\sqrt{h}}{8 \pi T} \delta\left[\square f_{R}(R)\right] .
\end{aligned}
$$

Now, we deal with terms of $\delta L_{\rho}$, respectively. Notice that the Tolman's law eq. (3.1) is used in our calculation. The first term of eq. (A.11) can be calculate as

$$
8 \pi \delta L_{\rho 1}=\frac{\sqrt{h}}{2 T} f_{R}(R) \delta R^{(3)}=\frac{1}{2} \chi \sqrt{h} f_{R}(R)\left(h^{a b} \delta R_{a b}^{(3)}+R_{a b}^{(3)} \delta h^{a b}\right) .
$$

The standard calculation of $\delta R_{a b}^{(3)}$ yields

$$
h^{a b} \delta R_{a b}^{(3)}=\sqrt{h} D^{a}\left(D^{b} \delta h_{a b}-h^{b c} D_{a} \delta h_{b c}\right),
$$

Using integration by parts and dropping boundary terms, we have

$$
\begin{aligned}
8 \pi \delta L_{\rho 1}= & \frac{1}{2} \sqrt{h} D^{b} D^{a}\left(\chi f_{R}(R)\right) \delta h_{a b}-\frac{1}{2} \sqrt{h} D_{c}\left[h^{a b} D^{c}\left(\chi f_{R}(R)\right)\right] \delta h_{a b} \\
& -\frac{1}{2} f_{R}(R) \chi \sqrt{h} R^{(3) a b} \delta h_{a b} .
\end{aligned}
$$


The third term of eq. (A.11) can be calculate as

$$
8 \pi \delta L_{\rho 3}=-\frac{\sqrt{h}}{T} \delta\left[u^{a} u^{b} \nabla_{a} \nabla_{b} f_{R}(R)\right]
$$

and the fourth term of eq. (A.11) can be written as

$$
8 \pi \delta L_{\rho 4}=-\frac{\sqrt{h}}{T} \delta\left[\square f^{\prime}(R)\right]=-\frac{\sqrt{h}}{T} \delta\left(\nabla_{a} \nabla^{a} f^{\prime}(R)\right) .
$$

Note that

$$
\begin{aligned}
D_{a} D^{a} f_{R}(R) & =h^{a b} \nabla_{a} \nabla_{b} f_{R}(R) \\
& =\nabla_{a} \nabla^{a} f_{R}(R)+u^{a} u^{b} \nabla_{a} \nabla_{b} f_{R}(R)
\end{aligned}
$$

and

$$
\delta\left(D_{a} D^{a} f_{R}(R)\right)=D_{a} \delta\left(D^{a} f_{R}(R)\right)+\delta C^{c}{ }_{a c} D^{a} f_{R}(R)
$$

where

$$
\delta C^{c}{ }_{a c}=\frac{1}{2} h^{c b} D_{a} \delta h_{c b} .
$$

So we have

$$
\begin{aligned}
8 \pi \delta L_{\rho 3} & +8 \pi \delta L_{\rho 4} \\
= & -\frac{\sqrt{h}}{T}\left[D_{a} \delta\left(D^{a} f_{R}(R)\right)+\delta C^{c}{ }_{a c} D^{a} f_{R}(R)\right] \\
= & -\sqrt{h} D^{a} \chi\left(D^{b} f_{R}(R)\right) \delta h_{a b}-\sqrt{h}\left(D^{a} D_{a} \chi\right) f_{R R}(R) \delta R \\
& +\frac{1}{2} \sqrt{h} h^{a b} D_{c}\left(\chi D^{c} f_{R}(R)\right) \delta h_{a b} .
\end{aligned}
$$

Considering the second term of eq. (A.11), together with eqs. (A.20) and (A.7), we denote all the terms contained $f_{R R}(R) \delta R$ by $\delta L_{\rho \delta R}$, then

$$
\begin{aligned}
8 \pi \delta L_{\rho \delta R} & =-\sqrt{h}\left(D^{a} D_{a} \chi\right) f_{R R}(R) \delta R+\frac{\sqrt{h}}{2} \chi\left(R^{(3)}-R\right) f_{R R} \delta R \\
& =-\sqrt{h}\left(D^{a} D_{a} \chi\right) f_{R R}(R) \delta R+\frac{\sqrt{h}}{2} \chi u^{a} u^{b} R_{a b} f_{R R} \delta R .
\end{aligned}
$$

We can calculate that

$$
\begin{aligned}
\chi R_{a b} u^{a} u^{b} & =R_{a c b}{ }^{c} u^{a} u^{b} \\
& =-\chi u^{a} \nabla_{a} \nabla_{c} u^{c}+\chi u^{a} \nabla_{c} \nabla_{a} u^{c} \\
& =\chi u^{a} \nabla_{c}\left(-u_{a} A^{c}\right)=\chi \nabla_{a} A^{a} .
\end{aligned}
$$

And we know

$$
\nabla_{a} A^{a}=A_{a} A^{a}+D_{a} A^{a} .
$$

So the $f_{R R}(R) \delta R$ terms vanish,

$$
\delta L_{\rho \delta R}=0
$$


At last, the calculation of $\delta \rho$ term of eq. (3.18) shows

$$
\begin{aligned}
\delta L_{\rho}= & \frac{\sqrt{h}}{16 \pi} D^{b} D^{a}\left(\chi f_{R}(R)\right) \delta h_{a b}-\frac{\sqrt{h}}{16 \pi} D_{c}\left[h^{a b} D^{c}\left(\chi f_{R}(R)\right)\right] \delta h_{a b}-\frac{\sqrt{h}}{16 \pi} f_{R}(R) \chi R^{(3) a b} \delta h_{a b} \\
& -\frac{\sqrt{h}}{8 \pi} \chi A^{a} D^{b} f_{R}(R) \delta h_{a b}-\frac{\sqrt{h}}{8 \pi} D^{a} \chi\left(D^{b} f_{R}(R)\right) \delta h_{a b}+\frac{\sqrt{h}}{16 \pi} h^{a b} D_{c}\left(\chi D^{c} f_{R}(R)\right) \delta h_{a b} \\
& +\frac{\sqrt{h}}{8 \pi} \chi A^{a}\left(D^{b} f_{R}(R)\right) \delta h_{a b} \\
= & \frac{\sqrt{h}}{16 \pi} D^{b} D^{a}\left(\chi f_{R}(R)\right) \delta h_{a b}-\frac{\sqrt{h}}{16 \pi} D_{c}\left[h^{a b} D^{c}\left(\chi f_{R}(R)\right)\right] \delta h_{a b}-\frac{\sqrt{h}}{16 \pi} f_{R}(R) \chi R^{(3) a b} \delta h_{a b} \\
& -\frac{\sqrt{h}}{8 \pi} D^{a} \chi\left(D^{b} f_{R}(R)\right) \delta h_{a b}+\frac{\sqrt{h}}{16 \pi} h^{a b} D_{c}\left(\chi D^{c} f_{R}(R)\right) \delta h_{a b} .
\end{aligned}
$$

This result tells us that the variation of $\rho$ term is exactly determined only by the variation of induced metric $\delta h_{a b}$.

\section{B Calculation of $P_{1}^{a b}$ and $P_{2}^{a b}$}

In this appendix, we give the detailed calculation of $P_{1}^{a b}$ and $P_{2}^{a b}$. We first calculate

$$
\begin{aligned}
P_{1}^{a b}= & \chi h^{a b}\left[f_{R}(R) R_{c d} u^{c} u^{d}+\frac{1}{2} f(R)-u^{c} u^{d} \nabla_{c} \nabla_{d} f_{R}(R)-\square f_{R}(R)\right] \\
& -\frac{1}{2} \chi f(R) h^{a b}+h^{a b} \chi \square f_{R}(R)-h^{a b} D_{c} D^{c}\left(\chi f_{R}(R)\right) \\
& +h^{a b} D_{c}\left(\chi D^{c} f_{R}(R)\right) \\
= & h^{a b}\left[\chi f_{R}(R) R_{c d} u^{c} u^{d}-\chi u^{c} u^{d} \nabla_{c} \nabla_{d} f_{R}(R)\right. \\
& \left.-D_{c} D^{c}\left(\chi f_{R}(R)\right)+D_{c}\left(\chi D^{c} f_{R}(R)\right)\right]
\end{aligned}
$$

In static spacetime, we have

$$
\begin{aligned}
& -\chi u^{c} u^{d} \nabla_{c} \nabla_{d} f_{R}(R) \\
& \quad=-\chi u^{c} \nabla_{c}\left[u^{d} \nabla_{d} f_{R}(R)\right]+\chi u^{c}\left(\nabla_{c} u^{d}\right) \nabla_{d} f_{R}(R) \\
& \quad=\chi A^{d} D_{d} f_{R}(R) .
\end{aligned}
$$

And notice eqs. (A.22) and (A.23),

$$
\begin{aligned}
\chi R_{c d} u^{c} u^{d} & =\chi \nabla_{c} A^{c} \\
& =\chi\left(A_{c} A^{c}+D_{c} A^{c}\right) .
\end{aligned}
$$

By substituting eqs. (B.2) and (B.3) in eq. (B.1),

$$
\begin{aligned}
P_{1}^{a b}= & h^{a b}\left[\chi\left(A_{c} A^{c}+D_{c} A^{c}\right) f_{R}(R)+\chi A^{d} D_{d} f_{R}(R)\right. \\
& \left.\quad-D_{c} D^{c}\left(\chi f_{R}(R)\right)+D_{c}\left(\chi D^{c} f_{R}(R)\right)\right] \\
= & h^{a b}\left[A^{c}\left(D_{c} \chi\right) f_{R}(R)+\chi\left(D_{c} A^{c}\right) f_{R}(R)-\left(D_{c} D^{c} \chi\right) f_{R}(R)\right] \\
= & 0 .
\end{aligned}
$$


Then, we calculate $P_{2}^{a b}$,

$$
\begin{aligned}
P_{2}^{a b}= & \chi\left[f_{R}(R) R_{c d} h^{a c} h^{b d}-h^{a c} h^{b d} \nabla_{c} \nabla_{d} f_{R}(R)\right]+D^{b} D^{a}\left(\chi f_{R}(R)\right) \\
& -f_{R}(R) \chi R^{(3) a b}-2 D^{a} \chi\left(D^{b} f_{R}(R)\right) .
\end{aligned}
$$

Applying eq. (A.8), we have

$$
\begin{aligned}
P_{2}^{a b}= & \chi f_{R}(R) R_{c d} h^{a c} h^{b d}-\chi D^{a} D^{b} f_{R}(R)+D^{a} D^{b}\left(\chi f_{R}(R)\right) \\
& -\chi f_{R}(R) h^{a c} h^{b d} R_{c d}-\chi f_{R}(R) R_{c e d}{ }^{l} u^{e} u^{l} h^{a c} h^{b d} \\
& -2 D^{a} \chi\left(D^{b} f_{R}(R)\right) \\
= & f_{R}(R)\left(D^{a} D^{b} \chi-\chi R_{c e d}{ }^{l} u^{e} u^{l} h^{a c} h^{b d}\right) .
\end{aligned}
$$

Using eq. (A.4), one can show that

$$
\begin{aligned}
R_{c e d}^{l} u^{e} u_{l} h^{a c} h^{b d} & =u^{e} h^{a c} h^{b d}\left(\nabla_{c} \nabla_{e} u_{d}-\nabla_{e} \nabla_{c} u_{d}\right) \\
& =u^{e} h^{a c} h^{b d}\left[\nabla_{c}\left(-u_{e} A_{d}\right)-\nabla_{e}\left(-u_{c} A_{d}\right)\right] \\
& =h^{a c} h^{b d}\left(\nabla_{c} A_{d}+A_{c} A_{d}\right)=D^{a} A^{b}+A^{a} A^{b},
\end{aligned}
$$

and

$$
\chi D^{a} A^{b}+\chi A^{a} A^{b}=D^{a} D^{b} \chi
$$

Hence,

$$
P_{2}^{a b}=0
$$

Open Access. This article is distributed under the terms of the Creative Commons Attribution License (CC-BY 4.0), which permits any use, distribution and reproduction in any medium, provided the original author(s) and source are credited.

\section{References}

[1] J.D. Bekenstein, Black holes and entropy, Phys. Rev. D 7 (1973) 2333 [InSPIRE].

[2] J.M. Bardeen, B. Carter and S.W. Hawking, The four laws of black hole mechanics, Commun. Math. Phys. 31 (1973) 161 [InSPIRE].

[3] S.W. Hawking, Particle creation by black holes, Commun. Math. Phys. 43 (1975) 199 [Erratum ibid. 46 (1976) 206] [INSPIRE].

[4] T. Jacobson, Thermodynamics of space-time: the Einstein equation of state, Phys. Rev. Lett. 75 (1995) 1260 [gr-qc/9504004] [INSPIRE].

[5] R.-G. Cai and S.P. Kim, First law of thermodynamics and Friedmann equations of Friedmann-Robertson-Walker universe, JHEP 02 (2005) 050 [hep-th/0501055] [INSPIRE].

[6] E.P. Verlinde, On the origin of gravity and the laws of Newton, JHEP 04 (2011) 029 [arXiv: 1001.0785] [INSPIRE].

[7] A. Bravetti, C.S. Lopez-Monsalvo and H. Quevedo, Maximally symmetric spacetimes emerging from thermodynamic fluctuations, arXiv: 1503.08358 [INSPIRE].

[8] W. J. Cocke, A maximum entropy principle in general relativity and the stability of fluid spheres, Ann. Inst. Henri Poincaré 2 (1965) 283. 
[9] R.D. Sorkin, R.M. Wald and Z.J. Zhang, Entropy of selfgravitating radiation, Gen. Rel. Grav. 13 (1981) 1127 [inSPIRE].

[10] S. Gao, A general maximum entropy principle for self-gravitating perfect fluid, Phys. Rev. D 84 (2011) 104023 [Addendum ibid. D 85 (2012) 027503] [arXiv:1109.2804] [INSPIRE].

[11] V. Iyer and R.M. Wald, Some properties of Noether charge and a proposal for dynamical black hole entropy, Phys. Rev. D 50 (1994) 846 [gr-qc/9403028] [INSPIRE].

[12] R.M. Wald, The thermodynamics of black holes, Living Rev. Rel. 4 (2001) 6 [gr-qc/9912119] [INSPIRE].

[13] S.R. Green, J.S. Schiffrin and R.M. Wald, Dynamic and thermodynamic stability of relativistic, perfect fluid stars, Class. Quant. Grav. 31 (2014) 035023 [arXiv:1309.0177] [INSPIRE].

[14] Z. Roupas, Thermodynamical instabilities of perfect fluid spheres in general relativity, Class. Quant. Grav. 30 (2013) 115018 [Corrigendum ibid. 32 (2015) 119501] [arXiv: 1411.0325] [INSPIRE].

[15] X. Fang and S. Gao, General proof of the entropy principle for self-gravitating fluid in static spacetimes, Phys. Rev. D 90 (2014) 044013 [arXiv:1311.6899] [InSPIRE].

[16] X. Fang and S. Gao, Proof of entropy principle in Einstein-Maxwell theory, Phys. Rev. D 92 (2015) 024044 [arXiv: 1506.01282] [INSPIRE].

[17] L.-M. Cao, J. Xu and Z. Zeng, Maximum entropy principle for self-gravitating perfect fluid in Lovelock gravity, Phys. Rev. D 87 (2013) 064005 [arXiv:1301.0895] [INSPIRE].

[18] L.-M. Cao and J. Xu, General proof of (maximum) entropy principle in Lovelock gravity, Phys. Rev. D 91 (2015) 044029 [arXiv:1404.6601] [INSPIRE].

[19] A.A. Starobinsky, A new type of isotropic cosmological models without singularity, Phys. Lett. B 91 (1980) 99 [INSPIRE].

[20] T.P. Sotiriou and V. Faraoni, $f(R)$ theories of gravity, Rev. Mod. Phys. 82 (2010) 451 [arXiv:0805.1726] [INSPIRE].

[21] A. De Felice and S. Tsujikawa, $f(R)$ theories, Living Rev. Rel. 13 (2010) 3 [arXiv: 1002.4928] [INSPIRE].

[22] A.V. Astashenok, S. Capozziello and S.D. Odintsov, Maximal neutron star mass and the resolution of the hyperon puzzle in modified gravity, Phys. Rev. D 89 (2014) 103509 [arXiv: 1401.4546] [INSPIRE].

[23] J.R. Oppenheimer and G.M. Volkoff, On massive neutron cores, Phys. Rev. 55 (1939) 374 [INSPIRE].

[24] R.M. Wald, General relativity, University of Chicago, Chicago U.S.A. (1984). 